\title{
Analysis of Warm Magnetic Measurements in a LHC Main Dipole Prototype
}

\author{
S. Gleis, J. Miles, O. Pagano, W. Scandale, E. Todesco. \\ CERN, LHC Division, CH-1211 Geneva 23, Switzerland
}

\begin{abstract}
The data relative to the magnetle measurements at room temperature of the MBP2N1 prototype of the LHC dipole are presented. The measured field harmonies are compared to the results of the numerical model of the dipole cross section. The contrbution from different effects are evaluated and compared to experimental data. Relevant quantlties on the mechanical structure and on the expected field quility are worked out.
\end{abstract}

\section{INTRODUCTION}

The 15-m long MBP2N1 LHC dipole prototype has a cross-section close to the one chosen for series production [1], i.e. 6-block coil design and collars without embedded magnetic insert, but featuring aluminum alloy collars (instead of austenitic steel ones) for reasons of component availability.

Magnetic measurements were performed at room temperature during the assembly and after the first poweritng tests. The results are used to study the field-shape performances of the dipole and to compare them with the expected values of the field harmonics computed with a magneto-static model [2]. References $[3,4]$ inspired our analysis of the field-shape quality.

After a short description of the main fealures of the hybrid prototype in Section II, we present the results of the magnetic measurements in Section III. In Section IV we discuss them in detail. First, we distinguish between systematic and random effects. Then, we analyze the multipole changes induced by the thermal and powering cycle. Finally, we itvestigate correlation between muttipoles measured at different stages of the dipole assembly. In Section $V$ we present our conclusions.

\section{Main Features of THE Dipole}

The 15-m long MBP2N1 prototype of the LHC main dipole features a cross-section close to the one chosen for series production, but for reasons of component availability, it is equipped with alumimum alloy collars instead of alstentic steel ones. For the same reasons, the geometry of the collars, inserts and laminations is not the finul one, thus making the contribution of the iron yoke to the dipole fieldshape different from the design one.

The coil presents a 6-block geometry; it consists of an inner and an outer layer, each wound with a keystoned cable, as shown in Fig. 1. Wedge-shaped copper spacers ane inserted between conductor blocks to correct the multipoles generated by the non-perfect $\operatorname{cosine}-\theta$ geometry. The coil layers are assembled into poles, which in turn are assembled into dipoles. Magnetic coupling between the two apertures is a feature of the two-in-one design, leading to the presence of even harmonics (quadrupole, octupole, decapole,...) in the dipole field. The contribution of the iron yoke enhances the strength of the magnet and limits the field-shape imperfections.

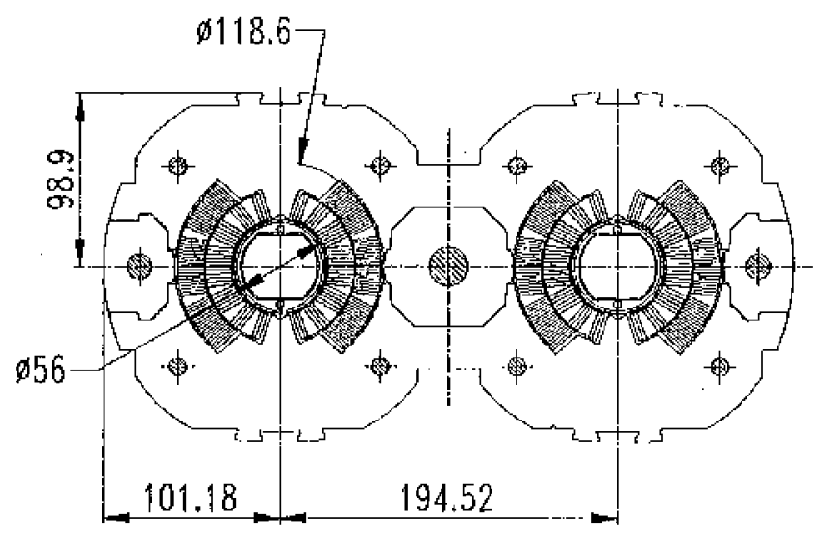

Fig. 1 Noninat design of the 6-blocka collared coil

\section{WARM MAGNETIC MEASUREMENTS}

The magnetic measurements are made at room temperature using a probe containing $0.75 \mathrm{~m}$ long rotating coils. The field shape is described in terms of relative multipoles expressed at a reference radius $R_{r e f}=17 \mathrm{~mm}$, in units of $10^{-4}$. At the selected excitation current of $20 \mathrm{~A}$, the measurement sensitivity is expected to be of the order of $10^{-7}$ units. The field-shape is megsured in both aperttres, at 20 consective positions along the dipole length. However, the first and the last measurements are discarded to avoid fringefield effects, since the first and the last positions include the dipole ends.

Measurements are performed on the collared coil, on the assembled cold mass and after the first cryo-powering test at high current. The results are summarized in terms of average and r.m.s. harmonics, computed over each aperture length. They are shown in Table I and II respectively.

During the meosurement process, field harmonics induce a feed-down effect to lower harmonics if the mechanical axis of the measurement coil is not coincident with the magnetic axis of the harmonics themselves. In general the largest induced hamonics are one order below the driving harnonic and they are linearly proportional to the mechanical offect between the magnetic axis and that of the measuring coil. The design of the 6-block coil is such that: 
- The allowed harmonics $b_{11}, b_{13}, b_{15}$, and $b_{17}$ are about a few $10^{-5}$ units at $17 \mathrm{ntm}$ reference radius.

- The non-allowed harmonics $b_{10}, b_{12}, b_{14}, b_{16}$ and $a_{10}$ $a_{12}, a_{14}, a_{16}$ are absent.

- Mechanical tolerances induce very small changes in the amplitude of harmonics greater than $10^{\text {th }}$ order.

TABLD I

MLAsURe MuLtTforts LA TrE MBP2N1 PROTOTYFE: A LNTS OF $10^{-4}$ AT $R_{\text {B日 }}=17 \mathrm{~mm}$.

\begin{tabular}{|c|c|c|c|c|c|c|}
\hline & \multicolumn{2}{|c|}{ Collared } & \multicolumn{2}{|c|}{ Assented } & \multicolumn{2}{|c|}{ Atter cryo } \\
\hline & Ap. 1 & $\mathrm{Ap} 2$ & Ap. 1 & Kp. 2 & $\mathrm{Ap}, 1$ & $A \mathrm{D} .2$ \\
\hline$a 2$ & 0.94 & 0.43 & 0.98 & 0.75 & 0.89 & 0.81 \\
\hline$b 2$ & -0.96 & 1.25 & -5.48 & 5.73 & -4.99 & 5.13 \\
\hline a3 & -0.11 & 0.29 & -0.38 & -0.01 & -0.46 & 0,00 \\
\hline$b 3$ & 2,08 & 2.71 & 8.09 & 8.68 & 8.17 & 8.71 \\
\hline$a 4$ & 0.06 & 0.05 & 0.05 & 0.10 & 0.07 & 0.11 \\
\hline 64 & $-0,07$ & 0.20 & 0.66 & 0.75 & -0.67 & 0.77 \\
\hline 05 & $-0,06$ & 0.05 & -0.07 & -0.02 & -0.08 & $-0,02$ \\
\hline b5 & $-0,63$ & -0.60 & -0.69 & -0.64 & -0.76 & $-0.7 \bar{k}$ \\
\hline 46 & 0.03 & 0.03 & 0.02 & 0.03 & 0.02 & 0.03 \\
\hline 06 & 0.00 & .0 .01 & -0.02 & 0.03 & -0.03 & 0.03 \\
\hline$a \gamma$ & 0.03 & 0,03 & 0.02 & 0.00 & 0.02 & 0.01 \\
\hline$b 7$ & 0.65 & 0.70 & 0.57 & $0.6 \mathrm{I}$ & 0.58 & 0.61 \\
\hline b9 & 0.25 & 0.26 & 0.26 & 0.26 & 0.21 & 0.20 \\
\hline bII & 0.73 & 0.73 & 0.63 & 0.62 & 0,63 & 0,62 \\
\hline
\end{tabular}

TABLE II

\begin{tabular}{|c|c|c|c|c|c|c|}
\hline & $\lambda_{p}, 1$ & Ap. 2 & Ap. 1 & $\mathrm{Ap} .2$ & $\lambda \mathrm{p} . \mathrm{T}$ & Ap. 2 \\
\hline 4 & 0.71 & 8,63 & 0.71 & 6.57 & 0.64 & 0.52 \\
\hline b2 & 0.36 & 0.43 & 0.39 & 0.44 & .40 & 0.46 \\
\hline a. & 0.44 & 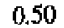 & 0. & 0. & I & 0.30 \\
\hline$b 3$ & 0.39 & 0. & 0. & 0.32 & 0.31 & 0.33 \\
\hline at & 0,26 & d) & 0 & 0.1 & 0.25 & 0.15 \\
\hline$b 4$ & 0.14 & 0 . & 0.12 & 0. & 0.12 & 0.11 \\
\hline 05 & 0,07 & 0.17 & 17 & 0.10 & 0.07 & 0.09 \\
\hline$b 5$ & 13 & & & 11 & 0.13 & 0.11 \\
\hline$a$ & 0.03 & & 0.03 & 0.03 & 0.02 & 0.03 \\
\hline bc & 0.08 & 0.06 & 0.07 & 0 & 0.07 & 0.05 \\
\hline$a 7$ & 0.02 & 0.15 & 0,02 & 0.02 & 0.02 & 0.02 \\
\hline 67 & 0,06 & 0.03 & 0.05 & 0.03 & 0.05 & 0.03 \\
\hline 69 & 0.01 & 0.01 & 0,01 & 0.02 & 0.02 & 0.02 \\
\hline bld & 0.00 & 0.0 & & 0.00 & 0,01 & 0.00 \\
\hline
\end{tabular}

It is natural to determine the local magnetic centre at different longitudinal positions in each apetture by assuning that the non-allowed harmonics are only due to first onder feed-down of odd normal harmonics according to:

$$
b_{2 n}=2 n b_{2 n+1} \frac{x_{0}(n)}{R_{r e f}} \quad a_{2 n}=2 n b_{2 n+1} \frac{y_{0}(n)}{R_{r e f}}
$$

where $n=5,6,7,8$. It follows that the magnetic centre $\left(x_{0}, y_{0}\right)$ is given by :

$$
x_{0}(n)=R_{\text {ref }} \frac{b_{2 n}}{2 n b_{2 n+1}} \quad y_{0}(n)=R_{r e f} \frac{a_{2 n}}{2 n b_{2 n+1}}
$$

We identified the offset between the axis of the rotating coil and the magnetic axis by applying these formulae to the feed down of $b_{17}$ in eacl aperulre and in each axial position and we slbtracted its effect in the results of Tables I and II.

\section{ANALYSIS OF THE DATA}

\section{A. Systematic Effects}

The data of Table I have been compared with the multipoles expected in the nominal collared coil, see Table III, and in the nominal assembled cold mass, see Table IV.

The simulations neglect the following contributions to the field-shape errors:

- Geometrical tolerances of the collars and coils.

- Deformations induced by mechanical stress during manufacturing.

TabLe III

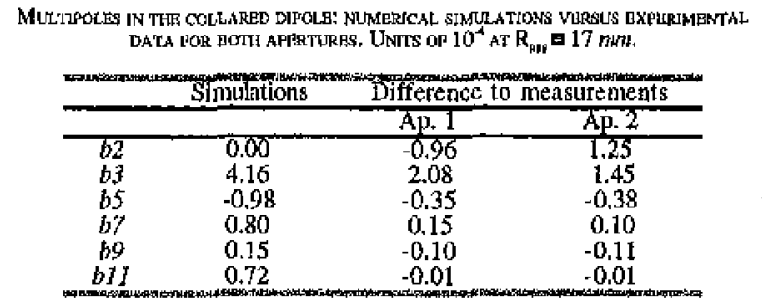

The first effect is the same for the collared coil and for the assembled cold nuass. The second one is in principle different since the yoke transmits an additional stress to the coils. In our case this fact onn be rather relevant since the collat is in aluminum. Indeed, the values of columns 3 and 4 in Table III are rather different from those in Table IV and it is not yet clear if this can be interpreted only in terms of the increased deformation induced by the yoking process. This discrepancy is at present the limit of out model for field-shape error estimates.

The simulated multipoles agree with the averages within \pm 3 standard deviations (see Tables II and III) of the measured data, with the only exceptions of $b_{4}$ and $b_{9}$. Corrective actions may be needed if this offset will persist and if it will appear to be detrinental for the circulating beant.

TAMTE IV

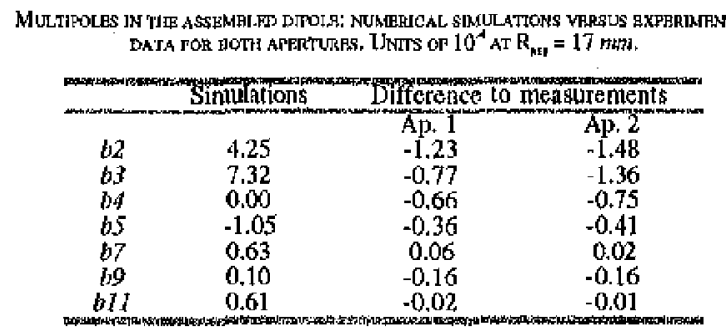




\section{B. Random Effects}

Random multipoles can be interpreted in terms of random displacement of the blocks. In Ref [5] it is shown that this interpretation gives rise to a very simple scaling law for the induced multipoles. The standard deviation $\sigma_{n}$ of the multipole tistribution of order $n$ scates linearly with the amplitude of the random displacement $d$ according to the following three-paraneter expression:

$$
\log _{10} \sigma_{n}=a+b n+c n^{2}
$$

or equivalently

$$
\sigma_{n}=A \cdot B^{n} \cdot C^{n^{2}}
$$

with $a, b$ and $c$ (or $A, B$ and $C$ ) independent of $d$. In addition, normal and skew components of the same order $n$ have ahout the same standard deviation $\sigma_{n}$.

The standard deviations of Table II can thus be compared to the predictions of the multipole scaling law, assuming independent block dtsplacements normally distributed, with zero mean and standard deviation $d$ along the dipole axis. In Fig. 2 the experimental data are compared to simulations obtained by varying $d$ from 6 to $100 \mu \mathrm{m}$. The observed multipoles are compatible with a value of $d$ in the ratige of 6 to $25 \mathrm{\mu m}$, such as in the previous 5-block coil prototypes [5]. This implies that the geometrically induced random multipoles are the same in the 5-block and in the 6-block coil design.

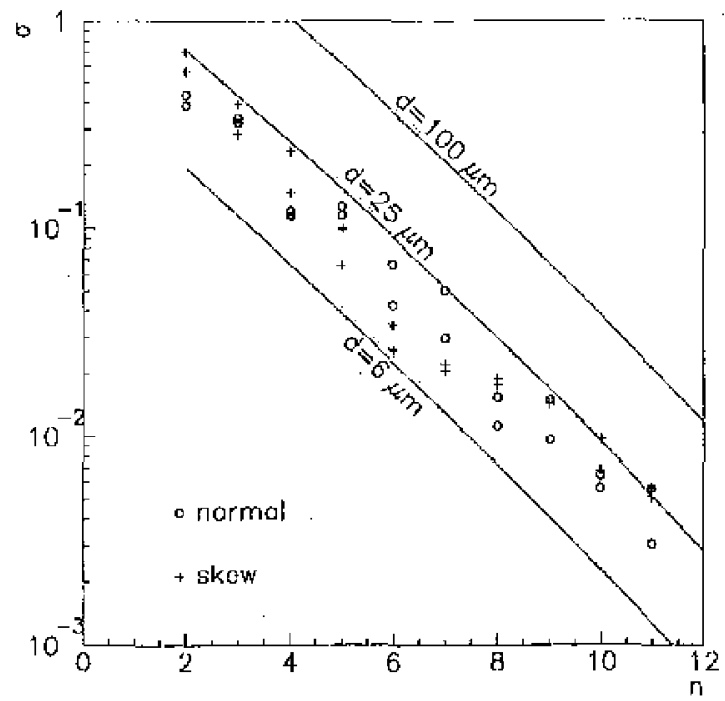

Fig, 2, Standard deviation of random toultipoles versus hamonic order $n$; Liprerimental data (dots) and mumerical simulations for different amplitudes of random displacoments $d$ (solid curves).

\section{Effect of Cycle to Cryogenic Temperature}

The shift of the average multipole harmonics measured at room temperature before the first cryogenic and powering test are shown in Table $V$. The effect is very small compared to that observed in the 5-block prototypes [5].

If we try to interptet it in terms of systematic movements of the blocks we find displacements of the order of a few tens of $\mu \mathrm{m}$, i.e. of the same order as the random displacements discussed in Stbsection $B$.

In comparison, coherent movements of about $50 \mu \mathrm{m}$ were deduced from the average multipole shifts observed in the 5 block coil prototypes [5]. This result confirms that the present 6-block coil design is mechanically more stable than the previous 5 -block coil design.

TAHLE V

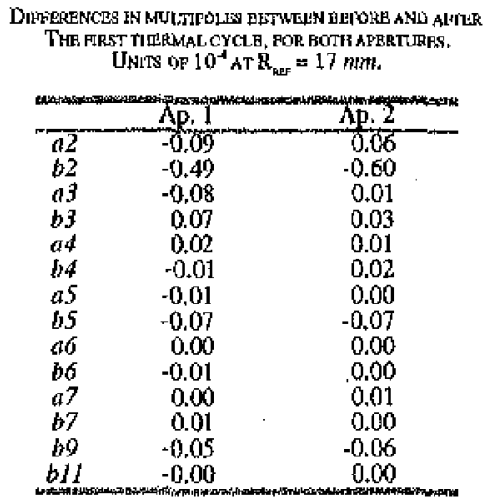

\section{Correlation between Mulipoles}

In Table VI we quote correlation between multipoles measured along the dipole axis, in the two apertures, for the collared coil and the cold mass. A linear law, as shown in Figs. 3,4 and 5 for the harmonics $b 3, b 5$ and $a 2$, respectively, very well interpolates experimental results. The coefficients $p$ and $q$ in Table VI are the slope and the ordinate at the origin of the interpolating line and $r^{*}$ is the correlation coefficient for the experimental data.

Tabile VI

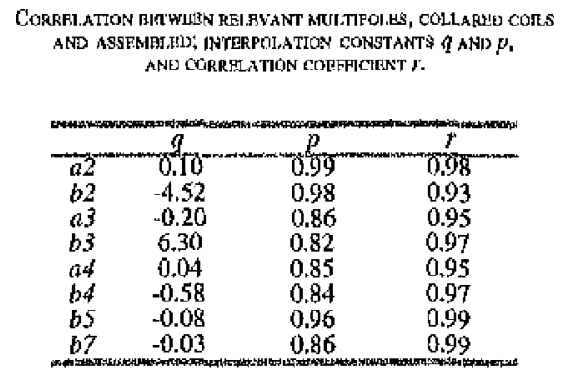


The slope $p$ is always close to one: this implies that the main effect of the yoke assembly is a shift of the hamonics that is particularly significant for $b_{2}, b_{3}$ and $b_{4}$.

We observe that the correlation is always excellent and that the harmonics have the same behavior in both apertures. This gives us confidence that measurements made in the collared coils give a reliable early indication of the fieldshape quality to be expected in the final cold mass. of course, this indication can be exploited to propose an economic and efficient quality control process during series production. The identification of corrective actions is still limited by the phenomenon discussed in Subsection $A$. Further studies in the final dipole design with stainless steel collars may clarify this point.

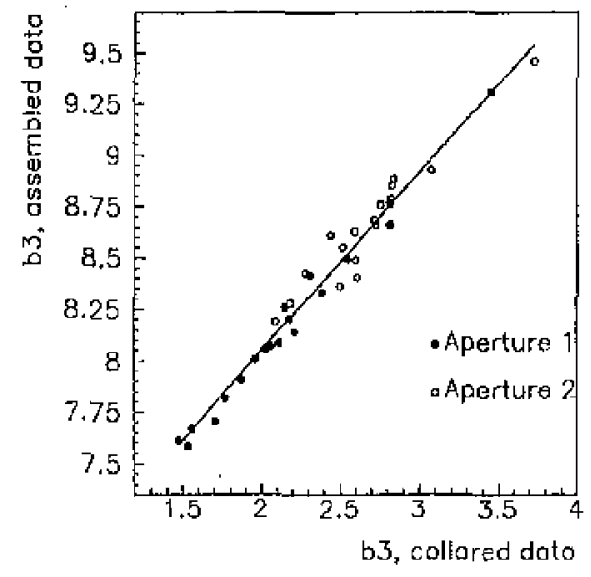

Fig. 3. Correlation of $b$, collared coll yersus assembled cold mass data (18 measurements along the dipole axis for both apertures). Units of $10^{4}$ at $\mathbf{R}_{\mathrm{ref}}=17 \mathrm{~mm}$

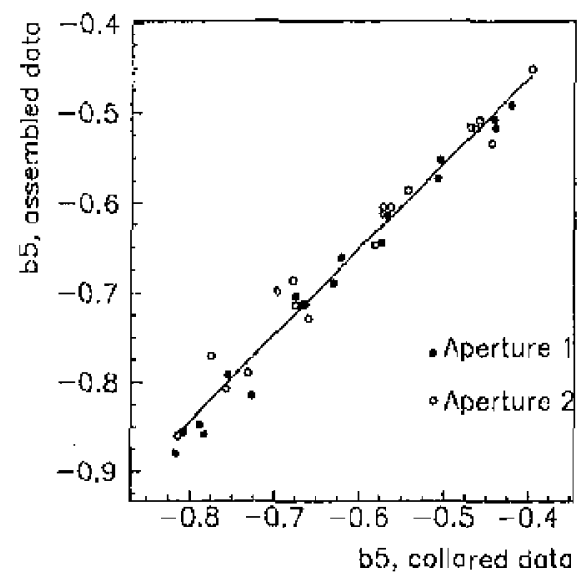

Fig. 4: Cotelation of $b$, collared coil versus assembled cold mass data (18 measurements along the dipole axis for both apertures). Units of $10^{-4}$ at $\mathrm{K}_{\mathrm{ret}} \neq 17 \mathrm{mtn}$

\section{CONCLUSION}

We discussed the magnetic measurements of the MBP2N1 dipole prototype. Although this magnet still differs from the final LHC dipole design, we could obtain numerous information that is expected to hold in general.

The order of magnitude of the systematic field-shape errors induced by geometrical imperfections is rather well predicted. The random variations of the multipoles along the dipole axis are quite limited and are induced by small geometrical misplacements of the conductors, characterized by a standard deviation in the range of 6 to $25 \mu \mathrm{m}$. In this respect the 5 - and 6 -block coil designs are identical. On the other hand, the coil deformation induced by the first cryogenic test of the dipole at high current is almost negligible. This conlirms that the 6-block design is very stable and almost immune to systematic block movements, contrary to what was previously observed in the 5-block coil [5].

The strong correlation observed in the field harmonics of the collared coll and of the fully assembled cold mass opens the way to an economic and efficient strategy of field-shape quality control during series production.

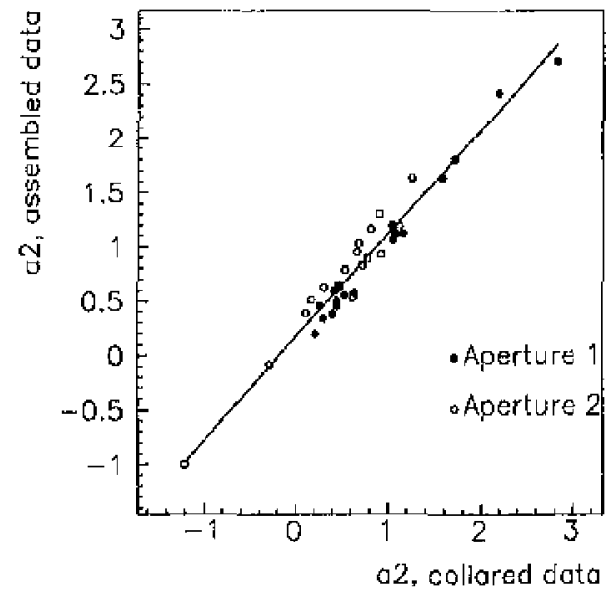

Fig. 5: Correlation of a2, collared coil versus assembled cold unass data ( 18 measurements along the dipole axis for both apertures). Units of $10^{-4}$ at $R_{\mathrm{rtf}}=17 \mathrm{~mm}$.

\section{REFERENCES}

[1] K. Artoos et al, Design, manufacturing status, fisst rosults of the LHC main dipole final prototypes and steps towards series munufacture, thefe Proc,

[2] S. Russenschuck, ed, CERN 09-01, 1999.

[3] R, Oupta et al, Field Quqlity Analysis as a Tool to Monitor Magnet Production, MT-15, Beijing, China, Ootober 20-24, 1997.

[4] I. Schmïser, DESY HERA-P, 92-1, 1992.

[5] O. Pagano, W. Scandale, E. Todesco, I.HC Project Note 180. 